\title{
Seismology of curved coronal loops with vertically polarised transverse oscillations
}

\author{
E. Verwichte, C. Foullon, and V. M. Nakariakov
}

\author{
Department of Physics, University of Warwick, Coventry CV4 7AL, UK \\ e-mail: Erwin.Verwichte@warwick . ac .uk
}

Received 28 October 2005 / Accepted 23 February 2006

\section{ABSTRACT}

\begin{abstract}
Aims. Using a model of vertically polarised fast magnetoacoustic waves in curved coronal loops, the method of coronal seismology is applied to observations of transverse loop oscillations.

Methods. A coronal loop is modeled as a curved magnetic slab in the zero plasma- $\beta$ limit. For an arbitrary piece-wise continuous power law equilibrium density profile, the dispersion relation governing linear vertically polarised fast magnetoacoustic kink waves is derived. The ways in which this model can be used for coronal seismology are explored and applied to two observational examples. Results. The Alfvén speed and equilibrium density profile are determined from observations. It is shown that the mechanism of lateral leakage of fast magnetoacoustic kink oscillations described in this model is efficient. In fact, the damping is so efficient that in order to match predicted values with observational ones, either the loop needs to be highly contrasted or the transverse Alfvén speed profile needs to be close to linear. Possible improvements to make the modeling of lateral wave leakage in loops more realistic, allowing a lower damping efficiency, are discussed.
\end{abstract}

Key words. Sun: oscillations - magnetohydrodynamics (MHD)

\section{Introduction}

The detection and identification of magnetohydrodynamic (MHD) waves and oscillations in the solar corona from recent observations using ground-based and space-borne instruments has made $M H D$ coronal seismology a practical, new tool for the determination of unknown parameters of the corona (see e.g. Nakariakov \& Verwichte 2005, for a review). The method of MHD coronal seismology was originally suggested by Uchida (1970) and Roberts et al. (1984). The technique is based upon measured properties of waves observed in a coronal structure on the one hand (e.g. period, wavelength, damping times) and a theoretical model describing waves in such structure on the other hand. By substituting the observed parameters into the model, we may determine the remaining unknown parameters that control the wave behaviour.

The technique of coronal seismology can be applied to various wave phenomena. Nakariakov et al. (2004) showed that the dispersive development of an impulsively generated fast magnetoacoustic wave pulse in a coronal loop (Roberts et al. 1984) leads to the formation of a quasi-periodic wave train, which has signatures that contain information about the transverse loop structuring. Similar signatures were found in eclipse observations (Katsiyannis et al. 2003), which strengthened the interpretation of the observed waves as fast magnetoacoustic wave trains. Robbrecht et al. (2001) and King et al. (2003) have shown that propagating slow magnetoacoustic waves observed co-spatially and co-temporally with multiple data sets, sensitive to different temperatures, point to a transverse temperature finestructuring of coronal loops.

Transverse oscillations of coronal loops (Aschwanden et al. 1999; Nakariakov et al. 1999) are of particular interest. These are interpreted as fast magnetoacoustic kink oscillations.
Nakariakov et al. (1999) and Nakariakov \& Ofman (2001) have shown that the Alfvén speed and the strength of the magnetic field of the coronal loop can be determined by substituting the observed oscillation period and wavelength (twice the loop length) of a fast magnetoacoustic kink oscillation into the straight loop model of Edwin \& Roberts (1983), and by assuming a realistic range of values for the loop density and contrast (see also Ofman \& Aschwanden 2002; Verwichte et al. 2004).

Coronal seismology is also expected to validate theoretical hypotheses put forward to explain certain aspects of observed wave behaviour. The observed rapid damping of fast kink oscillations (Nakariakov et al. 1999) cannot be explained by the direct dissipation caused by viscosity or resistivity, considering classical values of the shear viscosity and resistivity (see the discussion in Roberts 2000). Therefore, various alternative hypotheses have been put forward: e.g. resonant absorption with anomalous dissipation (Nakariakov et al. 1999), resonant mode conversion of quasi-modes (Ruderman \& Roberts 2002; Goossens et al. 2002) and phase-mixing with anomalous dissipation (Ofman \& Aschwanden 2002). We refer the reader to Nakariakov \& Verwichte (2005) for a discussion of these and other hypotheses. Recently, Brady \& Arber (2005) put forward the mechanism of lateral leakage by wave tunneling as an explanation for the rapid damping of vertically polarised kink oscillations. Ofman \& Aschwanden (2002) derived empirical scaling laws between observed parameters and concluded that phase mixing was the hypothesis that fitted best the observations, although resonant mode conversion could not be ruled out. In spite of this, Aschwanden et al. (2003) concluded in favour of the resonant mode conversion hypothesis (without ruling out phase mixing). The difference between the two studies is that in the former the ratio of the loop skin depth over width, a crucial parameter in the mechanism of resonant absorption, was not 
constrained. This example illustrates that for coronal seismology to be successful, all observed parameters need to be taken into account in a way as self-consistent as possible.

In Verwichte et al. (2006a) and Verwichte et al. (2006b), here after denoted Paper I and Paper II, an analytical model for vertically polarised fast magnetoacoustic waves in a curved coronal loop has been presented. We refer the reader to Paper I for a discussion of previous work on waves in curved coronal loops. The coronal loop has been modeled as a curved magnetic slab in the zero plasma- $\beta$ limit. The equilibrium density is given by a piecewise continuous power law profile. Depending on the value of the power law index, the wave modes are trapped or all subject to lateral wave leakage, either upwards or downwards. We have shown in Paper II that the theoretical model of upwards lateral wave leakage is consistent with the numerical simulations of Brady \& Arber (2005) and confirms that vertically polarised fast magnetoacoustic kink oscillations of isolated coronal loops may be efficiently damped due to lateral leakage. Furthermore, we have shown that fast kink oscillations in slender loops may have significant density perturbations, which is not the case in the straight loop model of Edwin \& Roberts (1982, 1983). However, fast kink may couple nonlinearly with slow magnetoacoustic modes which in turn produce substantial density perturbations. Terradas \& Ofman (2005) have shown that this mechanism may be responsible for observed intensity variations in transversely oscillating loops. Our theoretical model is well suited for coronal seismology as it predicts key wave observables that can be compared with observations, i.e. period, damping time and intensity amplitude. Here, we shall explore the potential of the model for coronal seismology using fast kink oscillations that experience upwards lateral wave leakage. We show that vertically polarised fast kink oscillations provide us with information of the transverse loop structuring.

The paper is structured as follows. In Sect. 2, the coronal loop model, the wave solutions and dispersion relation, used in Papers I and II, are briefly introduced. In Sect. 3, the intensity perturbations caused by a wave are discussed. In Sect. 4 , the ways in which the model can be used for coronal seismology are explored. In Sect. 5, two examples are given of the application of coronal seismology with the model to observations. In Sect. 6, the main findings, limitations and possible improvements of the model are discussed.

\section{Model and governing equations}

Following Papers I and II, the coronal loop is modeled as a semi-circular magnetic slab of half-width $a$ and radius of curvature $R$, in the gravitationless and zero plasma- $\beta$ limit (see Fig. 1 of Paper I for an illustration of the model). We use a cylindrical coordinate system with origin at the centre of curvature of the slab, at the solar surface. The slab and the equilibrium magnetic field are directed along the azimuthal $\phi$-direction, with the solar surface located at $\phi=[0, \pi]$. The solar surface is taken to be line-tied with rigid boundary conditions. The $z$ direction is parallel to the solar surface and perpendicular to the loop axis. In the radial direction with coordinate $r$, the semiinfinite space is partitioned into internal $(|r-R| \leq a)$ and external $(|r-R|>a)$ regions. Quantities in those regions are denoted with subscripts "i" and "e", respectively. The equilibrium magnetic field is azimuthal and of the form $B_{0 \phi}=B_{0}(r / R)^{-1}$. We choose the equilibrium density to be a piece-wise continuous power law, i.e. $\rho_{0}(r)=\rho_{0, \mathrm{i} / \mathrm{e}}(r / R)^{\alpha}$. Thus, the Alfvén speed is of the form $V_{\mathrm{A}}(r)=V_{\mathrm{Ai} / \mathrm{e}}(r / R)^{1-\delta}$, where $\delta=(\alpha+4) / 2$. The ratio $\chi=\rho_{0 \mathrm{e}} / \rho_{0 \mathrm{i}}$ is a measure of the density contrast and for a coronal loop this parameter is typically less than unity.

We consider vertically polarised fast magnetoacoustic kink oscillations in a loop with a density profile of index $\alpha>-4$, which covers the range of realistic density profiles. In Paper II, we have shown that for this case all waves leak out into the upper external medium and are therefore damped. We take the radial displacement, $\xi_{r}$, Eulerian magnetic pressure perturbation, $P$, and Eulerian density perturbation, $\rho$, of the fast kink oscillation to be of the form $f=\hat{f}(r) \sin (m \phi) \exp (-\mathrm{i} \omega t)$, where $\omega$ is the angular frequency of a mode of azimuthal degree $m$. Therefore, a wave of degree $m=1$ corresponds to the fundamental oscillation mode of the loop. Following Paper II, the solution of the relevant wave equation for a single mode of degree $m$ is given by

$$
\left(\frac{\hat{\xi}_{r}}{r}\right)= \begin{cases}A_{\mathrm{e}} \mathrm{J}_{v}(\sqrt{\chi} \Omega s) & r<R-a \\ A_{\mathrm{i}} \mathrm{J}_{v}(\Omega s)+B_{\mathrm{i}} \mathrm{Y}_{\nu}(\Omega s) & |r-R| \leq a \\ B_{\mathrm{e}} \mathrm{H}_{v}^{(1)}(\sqrt{\chi} \Omega s) & r>R+a\end{cases}
$$

where $s=(r / R)^{\delta} /|\delta|$ and $v=|m / \delta|$; the functions $\mathrm{J}_{v}, \mathrm{Y}_{v}$ and $\mathrm{H}_{v}^{(1)}$ are the Bessel, Neumann and first Hankel functions of order $v$; $\Omega$ is the dimensionless wave frequency $\Omega=\omega R / V_{\mathrm{Ai}}$. The wave period, $\mathrm{P}$, and damping time, $\tau$, are related to $\Omega$ with

$\mathrm{P}=\frac{2 \pi}{\mathfrak{R} \omega}=\frac{2 \pi}{\mathfrak{R} \Omega}\left(\frac{V_{\mathrm{Ai}}}{R}\right)^{-1}$,

and

$\tau=-\frac{1}{\mathfrak{J} \omega}=-\frac{1}{\mathfrak{I} \Omega}\left(\frac{V_{\mathrm{Ai}}}{R}\right)^{-1}$

As in Paper I, the magnetic pressure is calculated using

$\left(\frac{\hat{P}}{P_{0}}\right)=-2 r \frac{\mathrm{d}}{\mathrm{d} r}\left(\frac{\hat{\xi}_{r}}{r}\right)$

$P_{0}$ is the equilibrium magnetic pressure. The continuity of the displacement, $\hat{\xi}_{r}$, and the Lagrangian total pressure perturbation, $\delta \hat{P}=\hat{P}-2 P_{0} \hat{\xi}_{r} / r$, at $r=R \pm a$ leads to the dispersion relation

$\frac{\mathcal{W}\left\{\mathrm{J}_{v}\left(\Omega s_{-}\right), \mathrm{J}_{v}\left(\sqrt{\chi} \Omega s_{-}\right)\right\}}{\mathcal{W}\left\{\mathrm{Y}_{v}\left(\Omega s_{-}\right), \mathrm{J}_{v}\left(\sqrt{\chi} \Omega s_{-}\right)\right\}}=\frac{\mathcal{W}\left\{\mathrm{J}_{v}\left(\Omega s_{+}\right), \mathrm{H}_{v}^{(1)}\left(\sqrt{\chi} \Omega s_{+}\right)\right\}}{\mathcal{W}\left\{\mathrm{Y}_{v}\left(\Omega s_{+}\right), \mathrm{H}_{v}^{(1)}\left(\sqrt{\chi} \Omega s_{+}\right)\right\}}$,

where $\mathcal{W}\{f(x), g(y)\}=f(x) \frac{\mathrm{d} g}{\mathrm{~d} r}(y)-\frac{\mathrm{d} f}{\mathrm{~d} r}(x) g(y)$ and $s_{ \pm}=(1 \pm$ $a / R)^{\delta} /|\delta|$. Equation (5) is solved for $\Omega$ as a function of $m, a / R$, $\chi$ and $\alpha$. The value of $V_{\mathrm{Ai}}$ only enters into the model through Eqs. (2) and (3).

\section{Intensity perturbation}

In Papers I and II we have shown that the vertically polarised kink mode is compressible in a curved loop. Hence, it perturbs the loop density. Therefore, besides the period and damping time, the intensity perturbation relative to the displacement is an additional observable, which may contain information about the loop. For resonant EUV line emission in the optically thin corona, the intensity relates to the density as $I \sim \rho^{2}$. The Lagrangian intensity perturbation, $\delta I$, is, for small amplitudes, equal to $2 \rho_{0} \delta \rho$. Here, $\delta \rho$ is the Lagrangian density perturbation given by (see Paper I)

$\left(\frac{\delta \hat{\rho}}{\rho_{0}}\right)=\frac{\hat{\rho}}{\rho_{0}}+\frac{1}{\rho_{0}} \hat{\xi}_{r} \frac{\mathrm{d} \rho_{0}}{\mathrm{~d} r}=\frac{1}{2} \frac{\hat{P}}{P_{0}}-2 \frac{\hat{\xi_{r}}}{r}$ 

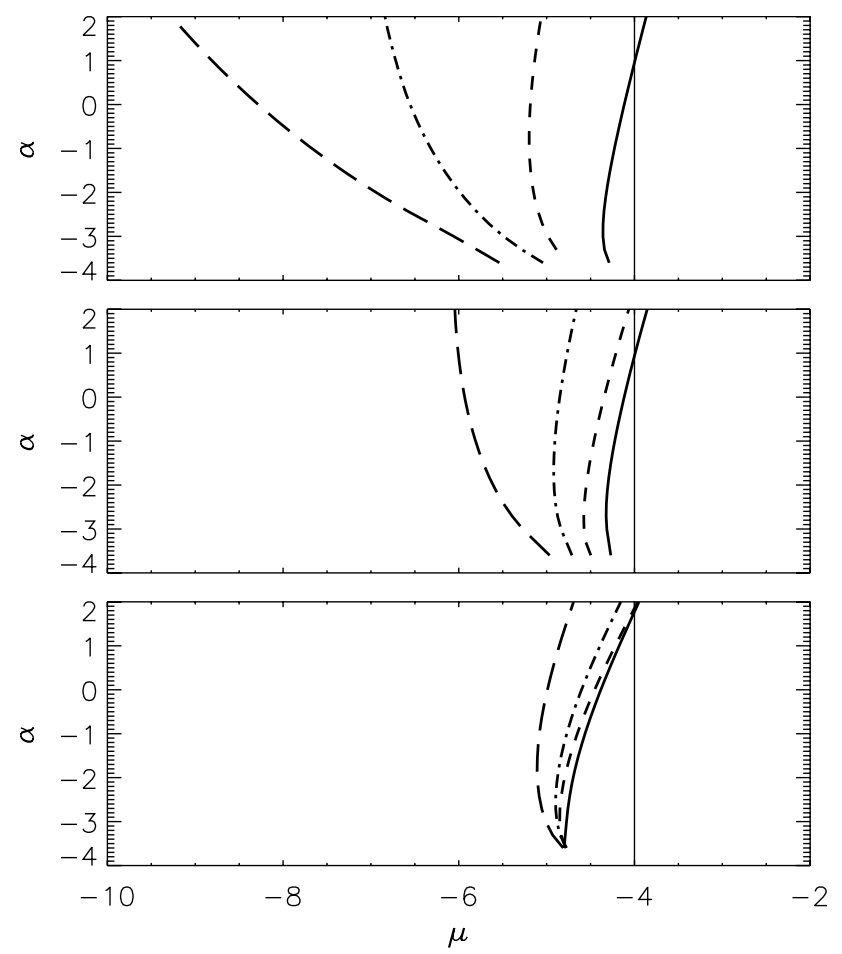

Fig. 1. Equilibrium density power law index $\alpha$ as a function of the amplitude ratio $\mu$ at the loop top of the fast kink mode of degree $m=1$, for three values of $a / R$ : 0.01 (top), 0.05 (middle), 0.2 (bottom). The curves represent results for different values of $\chi: 0.01$ (solid), 0.1 (dashed), 0.25 (dot-dashed) and 0.5 (long-dashed). The thin solid line is the approximate value of $\mu$ using Eq. (9).

It is convenient to introduce here the ratio between the average intensity perturbation amplitude and the average loop displacement as

$\mu=\frac{\langle\delta I\rangle}{\left\langle I_{0}\right\rangle\left\langle\xi_{r} / r\right\rangle}$,

where $I_{0}$ is the equilibrium intensity. The averaging operator \langle\rangle is defined as

$\langle f\rangle=\frac{1}{2 a} \int_{R-a}^{R+a} f(r) \mathrm{d} r$,

where $f$ is a linear perturbation. Averaging explicitly over the perturbed interval gives approximately the same result.

We calculate $\mu$ for a fast kink mode of degree $m=1$ using Eq. (7) as a function of $\alpha$ for a range of values in $a / R$ and $\chi$ (solving dispersion relation (5) and calculating the Lagrangian density perturbation and displacement using Eqs. (1) and (6) respectively). The result is shown in Fig. 1. The fast kink mode of degree $m=1$ is characterised by a radial translation of the whole loop in the same direction. In Paper I we argued, on physical grounds without explicit calculations, that the ratio of the relative average intensity perturbation and the displacement amplitude for such a mode is approximately

$\mu=2 \frac{\left\langle\rho_{0} \delta \rho\right\rangle}{\left\langle\rho_{0}^{2}\right\rangle\left\langle\xi_{r} / r\right\rangle} \approx \frac{\left\langle P / P_{0}\right\rangle}{\xi_{r}(R) / R}-4 \approx-4$,

where $\left\langle P / P_{0}\right\rangle$ was assumed to be small, as is the case with the fast kink mode in a straight loop model. Equation (9) suggests that the ratio of the relative intensity perturbation and the displacement amplitude is independent of the unknown parameters and is equal to -4 . This would imply that the intensity variation does not provide additional information that can be used for seismological purposes. However, Fig. 1 shows that Eq. (9) overestimates the value of $\mu$ because the contribution of the average Eulerian total pressure perturbation cannot be neglected. The largest departures from Eq. (9) occur for faint (large $\chi$ ), slender (small $a / R$ ) loops. Also, the result does not change significantly if $\left\langle\xi_{r} / r\right\rangle$ is replaced by $\xi(R) / R$. The quantity $\mu$ is a function of $\alpha$, $\chi$ and $a / R$, and can therefore be used for seismological purposes after all. However, the index $\alpha$ cannot be determined from the intensity variation independently from $\chi$ and $a / R$. Also, the calculation of $\mu$ does not involve the value of $V_{\mathrm{Ai}}$.

Even if the displacement of the loop is less than the resolution of an imaging instrument such as the Transition Region And Coronal Explorer (TRACE), a vertically polarised kink loop oscillation may still be detected as intensity oscillations. For example, a loop of length $50 \mathrm{Mm}$, which has a vertical displacement corrresponding to the distance seen by a TRACE pixel, i.e. $0.35 \mathrm{Mm}$, has a minimum relative absolute intensity perturbation of $3 \%$ according to Eq. (9). Furthermore, the intensity variations of a fundamental kink oscillation are most pronounced near the loop top and can therefore be distinguish from the fundamental slow magnetoacoustic oscillation, which has intensity variations near the loop footpoints.

To get $\mu$ from the observations, it is important to take into account the effect of time-integration, because a transversely oscillating loop, with a component of displacement in the plane of the sky, will contribute longer to the pixel intensity at the extreme loop positions than in between. When considering comparisons with our theoretical model, this effect can be corrected by normalising the measured intensity with the time-integrated intensity of an oscillating, homogeneous loop of the same width. Unfortunately, this correction is missing in the currently known observational example (Wang \& Solanki 2004). Thus, the effective application of $\mu$ of vertically polarised kink modes for purposes of coronal seismology awaits a reanalysis of this example or the discovery of new ones.

\section{Determination of loop transverse parameters}

\subsection{General method}

The above theoretical model can be compared to observations of vertically polarised fast magnetoacoustic kink oscillations in coronal loops, and may therefore be used for seismological purposes. From observations, the following wave signatures can be measured: oscillation period, $\mathrm{P}$, damping time, $\tau$, azimuthal degree, $m$, loop axis displacement amplitude, $\xi_{r}(R) / R$ and the relative average intensity perturbation, $\langle\delta I\rangle /\left\langle I_{0}\right\rangle$. Also from the observations, the loop half-width, $a$, and loop radius of curvature, $R$, can be estimated. The latter quantity is obtained by fitting the loop shape with a semi-circle. This assumption, together with projection effects, introduces a relative error in $R$, which may be estimated qualitatively to be at least $10 \%$ (e.g. Aschwanden et al. 2002).

The theoretical model needs three additional parameters to fully describe the wave behaviour: e.g. the equilibrium density power law index, $\alpha$, the density contrast, $\chi$ and the internal Alfvén speed, $V_{\mathrm{Ai}}$. Through $V_{\mathrm{Ai}}$ and $\chi$, the external Alfvén speed, $V_{\mathrm{Ae}}$, is determined. The parameters $\chi$ and $\alpha$, which are connected with the transverse loop structuring, may be measured directly from observations, but the narrowness of EUV instrument temperature bandpasses and problems with background subtraction due to line-of-sight effects introduce large 

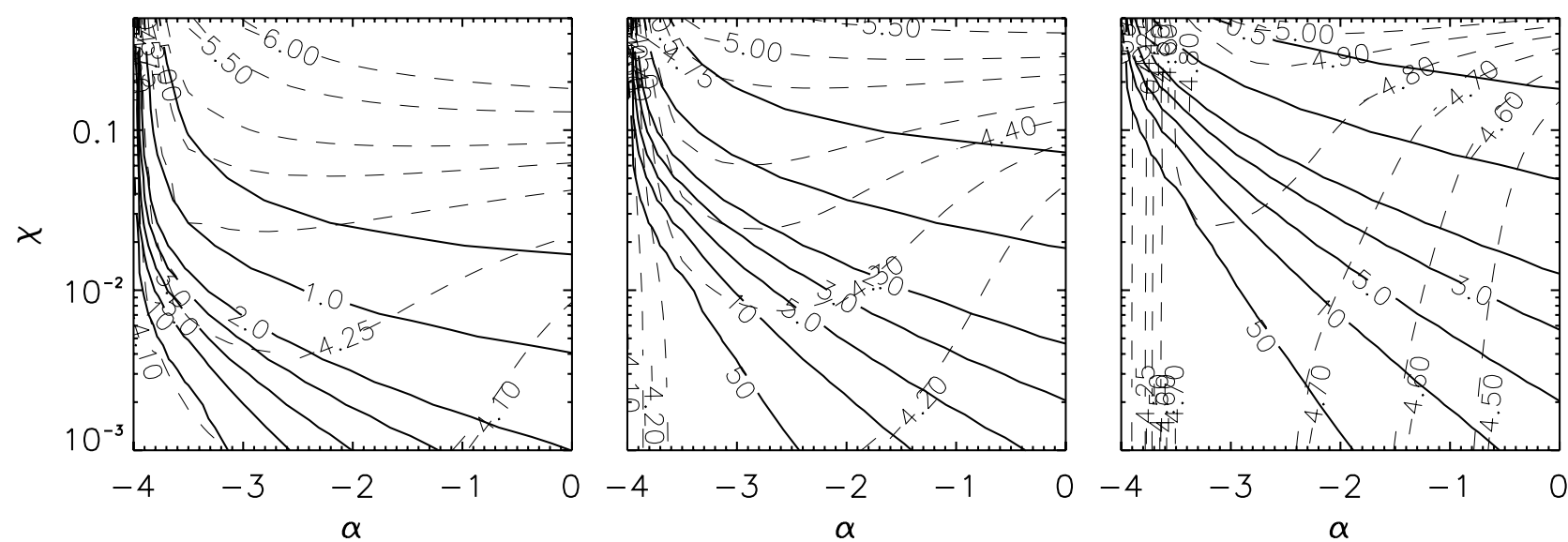

Fig. 2. Parameter space $\alpha-\chi$ for three values of $a / R$ : 0.01 (left), 0.05 (middle) and 0.2 (right). The solid and dashed curves represent constant values of $\tau / \mathrm{P}$ and $\mu$, respectively.
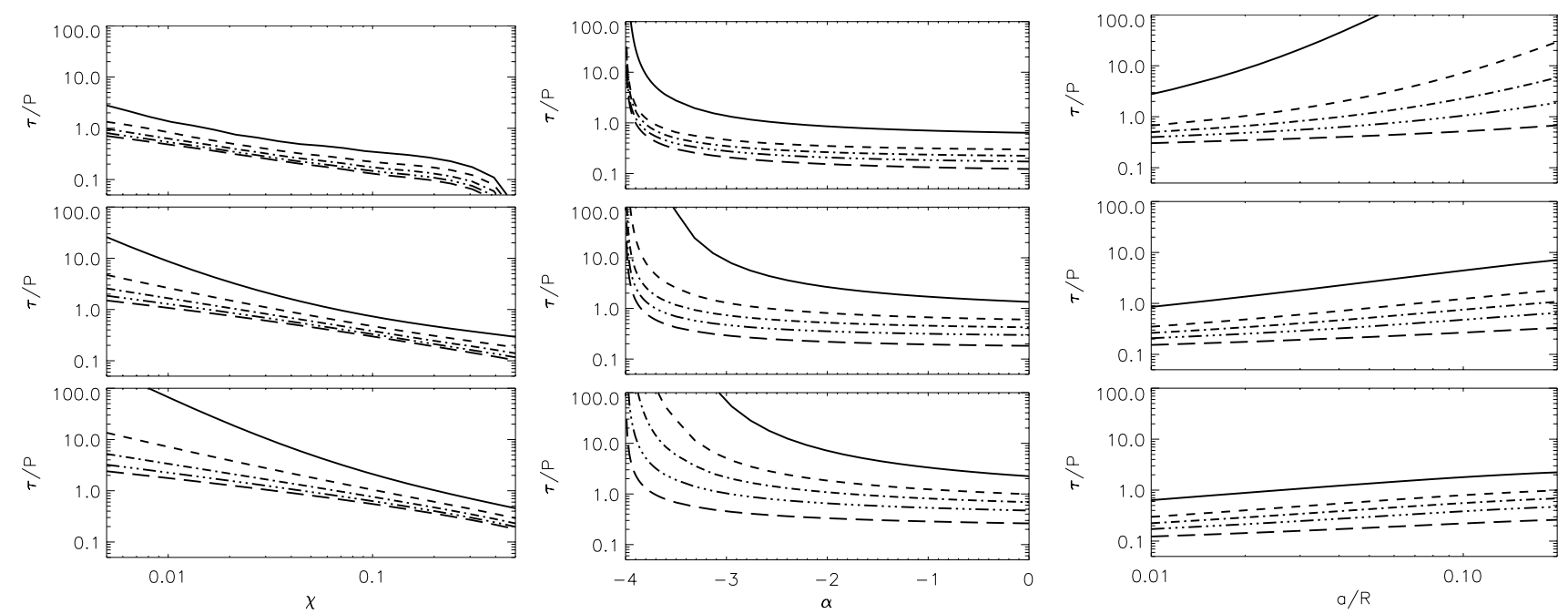

Fig. 3. (Left) Quality factor $\tau / \mathrm{P}$ as a function of $\chi$ for three values of $a / R: 0.01$ (top), 0.05 (middle) and 0.2 (bottom). The curves represent different values of $\alpha$ : -3 (solid), -2 (dashed), -1 (dot-dashed), 0 (triple dot-dashed) and 1 (long-dashed). (Middle) Quality factor $\tau / \mathrm{P}$ as a function of $\alpha$ for three values of $a / R: 0.01$ (top), 0.05 (middle) and 0.2 (bottom). The curves represent different values of $\chi: 0.01$ (solid), 0.05 (dashed), 0.1 (dot-dashed), 0.2 (triple dot-dashed) and 0.5 (long-dashed). (Right) Quality factor $\tau / \mathrm{P}$ as a function of $a / R$ for three values of $\alpha$ : -3.5 (top), -2 (middle) and 0 (bottom). The curves represent different values of $\chi$ : 0.01 (solid), 0.05 (dashed), 0.1 (dot-dashed), 0.2 (triple dot-dashed) and 0.5 (long-dashed).

uncertainties (e.g. Aschwanden et al. 2003). Therefore, we propose to use the observed wave signatures to determine these parameters.

In mathematical terms, the method proposed here involves a system of equations in the three unknown parameters, which relate the observed parameter values to their theoretically predicted values. By solving dispersion relation (5), we can calculate theoretical predictions of $\mu$ using Eq. (7), P using Eq. (2) and $\tau$ using Eq. (3), as a function of the unknown parameters $\alpha$, $V_{\mathrm{Ai}}$ and $\chi$ and the known fixed values of $m$ and $a / R$. Thus, we create a three-dimensional space in the unknown parameters. Each of the three relations between the predicted and the observed value of a parameter forms a surface in this space. Where the three surfaces cross a set of values for $\alpha, V_{\mathrm{Ai}}$ and $\chi$ is found that is consistent with the model and the observations. This is an exactly determined system. If one of the parameters is already known, then the system becomes overdetermined. We can then verify the applicability of the model and its underlying assumptions.

However, the three-dimensional parameter space does not need to be studied in full if we note that the quantities $\mu$ and $\tau / \mathrm{P}$ do not depend on $V_{\mathrm{Ai}}$. The ratio $\tau / \mathrm{P}$ is known as the quality factor. We can construct a two-dimensional parameter space in $\alpha$ and $\chi$ in which the relations between the predicted and observed values of $\tau / \mathrm{P}$ and $\mu$ form two curves. Where the two curves cross, a set of values for $\alpha$ and $\chi$ is found that is consistent with theory and observations. With $\alpha$ and $\chi$ determined, the shape of the density profile is fixed and all parameters for calculating the dimensionless mode frequency using Eq. (5) are known. Along each curve, the value of the Alfvén speed varies.

The behaviour of the curves of constant $\tau / \mathrm{P}$ and $\mu$ in the $\alpha-\chi$ parameter space is shown in Fig. 2. On curves of constant $\tau / \mathrm{P}, \chi$ increase as $\alpha$ becomes smaller. From the point of view of a tunneling mode for instance, this can be understood as follows. As $\alpha$ decreases, the evanescent barrier through which the wave tunnels becomes thicker. Therefore, the density contrast has to be lowered (increasing $\chi$ ) to maintain the same damping rate. The dependencies of the quality factor on $\chi$ and $\alpha$ respectively, are shown in Fig. 3 (left and middle panels). Figures 1 and 2 show that $\mu$ becomes independent of $\chi$ as $\alpha$ approaches -4 . Also, these curves show a minimum as a function of $\alpha$. Therefore, it is possible to find more than one crossing between a particular 
set of curves of constant $\tau / \mathrm{P}$ and constant $\mu$, and hence multiple solutions for $\alpha$ and $\chi$. In that case, additional arguments are needed for favouring one solution above another.

If the system is overdetermined, then the applicability of the physical mechanism in the model can be tested. Ofman \& Aschwanden (2002) compared theoretical predictions with observations using scaling laws between $\tau$ and P. However, for most models, this requires knowledge of the Alfvén speed. The quality factor is therefore better suited. From Fig. 3 we see that $\tau / \mathrm{P}$ decreases with increasing $\chi, \alpha$ or $R$. This sets our model apart from other mechanisms put forward to explain the damping of transverse loop oscillations. Indeed, the mechanism of resonant mode conversion of quasi-modes predicts that $\tau / P$ increases with increasing $\chi$ and is independent of $R$ (Ruderman \& Roberts 2002); the mechanism of phase-mixing with anomalous dissipation predicts that $\tau / \mathrm{P}$ decreases with increasing $R$ but is independent of $\chi$ (Ofman \& Aschwanden 2002).

\subsection{Determination of the Alfvén speed}

Nakariakov et al. (1999) and Nakariakov \& Ofman (2001) have shown how the Alfvén speed in a coronal loop can be determined from the observed oscillation period of a fast kink mode of degree $m=1$ and the loop length. They applied the model of a straight cylinder loop with piece-wise homogeneous equilibrium quantities and used the fact that the phase speed of the fast kink mode is equal to the kink speed in the long wavelength limit, independent of the value of $a / R$ (Edwin \& Roberts 1983). By making some assumptions for the value of the density contrast, the Alfvén speed can then be determined from the kink speed. In a straight magnetic slab model (Edwin \& Roberts 1982), the phase speed of the fast kink mode tends to the external Alfvén speed in the long wavelength limit. Again, the density contrast is needed to relate the external to the internal Alfvén speed.

In our model, the phase speed becomes infinite in the long wavelength limit. Therefore, the phase speed of a fast kink mode needs to be calculated explicitly for given values of the parameters $\alpha, \chi$ (determined by the method outlined above), $a$ and $R$. The internal Alfvén speed is then derived from Eq. (2). Figure 4 shows this process graphically: if all loop parameters are known, then from the observed oscillation period, the internal Alfvén speed can be determined from Fig. 4. Note, that with $\mathrm{P}$ and $R$ fixed, $V_{\mathrm{Ai}}$ increases with decreasing $\alpha$. Also, $V_{\mathrm{Ai}}$ depends weakly on $\alpha$ for highly contrasted loops because the more contrasted a coronal loop is, the more it behaves independently from the external medium and its density profile.

\section{Examples}

Here, we shall study two observational examples of fast magnetoacoustic kink oscillations in coronal loops observed by TRACE. For this, we assume that the observed oscillation damping is a manifestation of lateral wave leakage as studied in Paper II. In both cases $\mathrm{P}$ and $\tau$ are measured. Unfortunately, no reliable measurements of $\mu$ currently exist. In the first example, $\alpha$, $V_{\mathrm{Ai}}$ and $\chi$ are all unknown. Therefore, we have an underdetermined system. In the second example, $\alpha$ and $V_{\mathrm{Ai}}$ are unknown but $\chi$ is measured, so that we have an exactly determined system in two parameters.
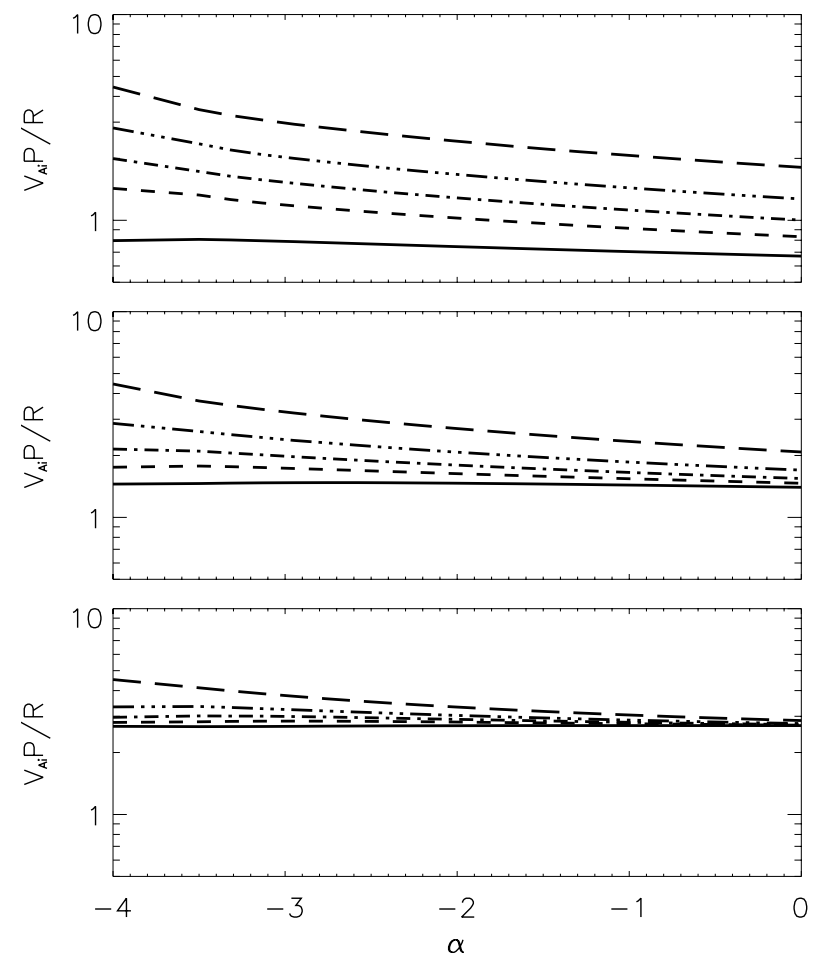

Fig. 4. Normalised wave period, $V_{\mathrm{Ai}} P / R$ as a function of $\alpha$ for three values of $a / R$ : 0.01 (top), 0.05 (middle) and 0.2 (bottom). The curves represent different values of $\chi$ : 0.01 (solid), 0.05 (dashed), 0.1 (dotdashed), 0.25 (triple dot-dashed) and 0.5 (long-dashed).

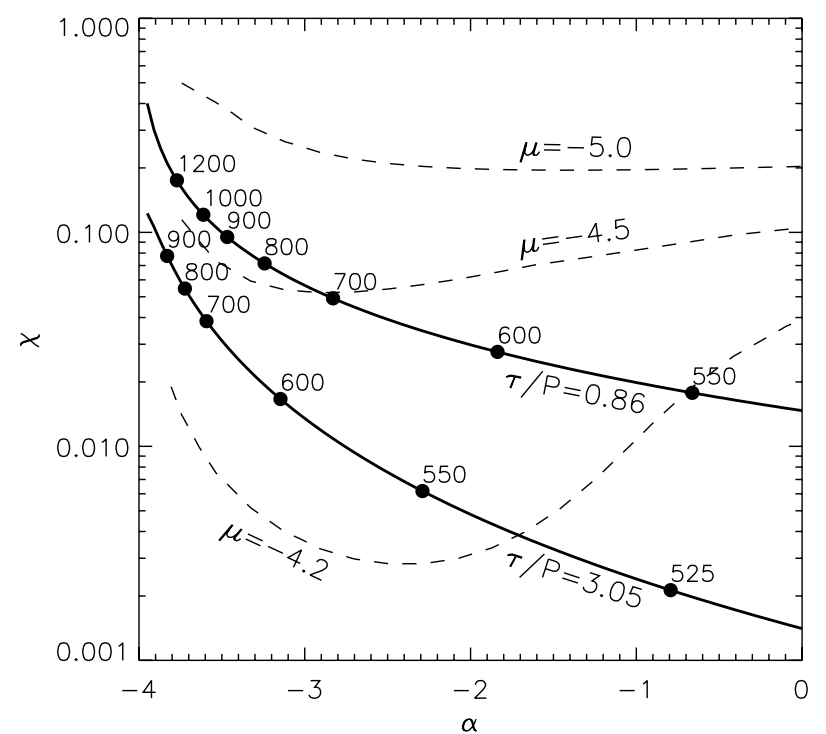

Fig. 5. Parameter space $\alpha-\chi$ for example 1. The solid curves represent constant quality factor $\tau / \mathrm{P}$, calculated using solution of Eq. (5) and matching the observed values of $3.05(\tau=714 \mathrm{~s})$ or $0.85(\tau=200 \mathrm{~s})$. The aspect ratio $a / R=0.03$. Along these curves the value of $V_{\mathrm{Ai}}$ varies monotonically, as indicated by key values of $V_{\mathrm{Ai}}$ in units of $\mathrm{kms}^{-1}$ are indicated. The dashed curves represent constant values of $\mu$.

\subsection{Example 1}

We consider the only existing observation of a vertically polarised fast kink oscillation to date, reported by Wang \& Solanki (2004). The following values of the oscillating coronal loop were determined: $R=112(95 ; 127) \mathrm{Mm}, m=1, \mathrm{P}=234 \mathrm{~s}$, $\tau=714 \mathrm{~s}, \xi_{r}(R)=7.9 \mathrm{Mm}$ and $\langle\delta I\rangle /\left\langle I_{0}\right\rangle=-1.0$. Here and in the following, the range is given between brackets. After 

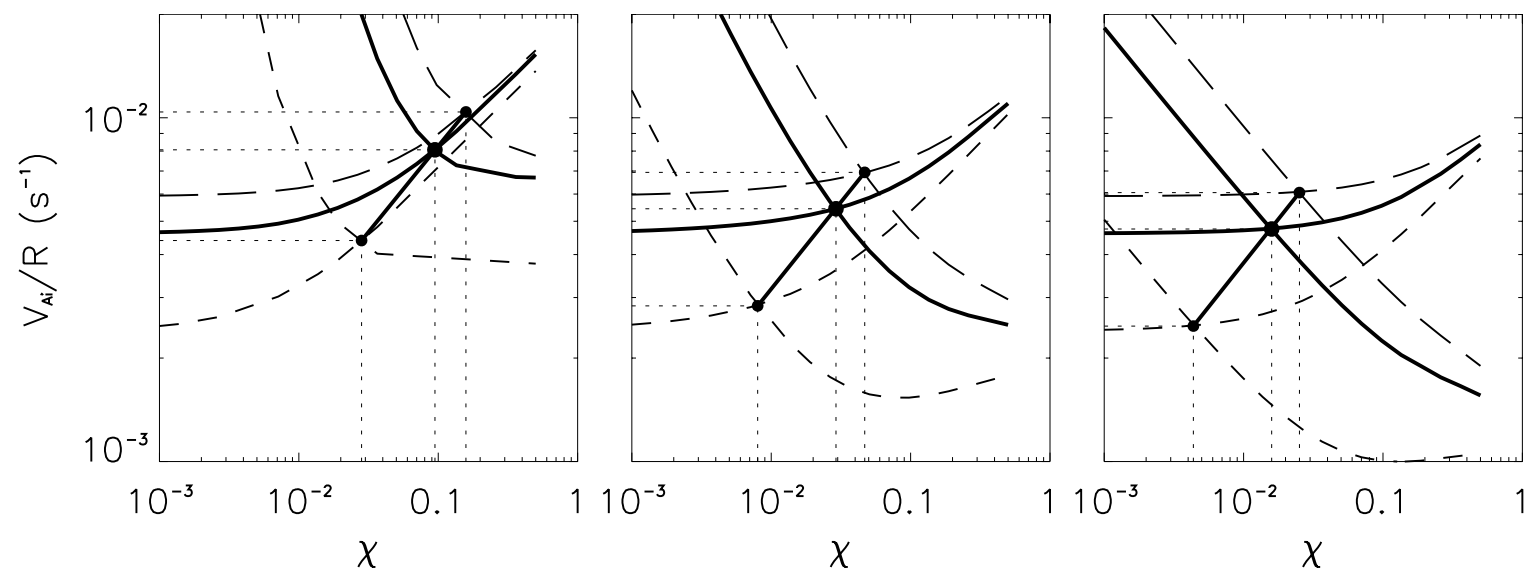

Fig. 6. Ratio $V_{\mathrm{Ai}} / R$ as a function of $\chi$ for the fast kink mode of degree $m=1$, for three values of $a / R: 0.008$ (dashed), 0.03 (solid) and 0.05 (long dashed). Each pair of curves is calculated using the observed oscillation period, $\mathrm{P}=234 \mathrm{~s}$, and damping time, $\tau=200 \mathrm{~s}$, respectively. The crossing of the pair of curves indicates a value for $V_{\mathrm{Ai}} / R$ and $\chi$ consistent with the model and the observations. Left: $\alpha=-3.5$. Middle: $\alpha=-2$. Right: $\alpha=0$.

visual inspection of Fig. 2 of Wang \& Solanki (2004), we measure $a=3.6(1 ; 5) \mathrm{Mm}$, giving a generous range of values for $a$. The ratio $a / R$ is then equal to $0.03(0.008 ; 0.05)$, with a relative range of about $70 \%$. When dealing directly with the data, $a$ can be determined more precisely. Because only 1.5 oscillation periods of the oscillation have been observed due to a data gap, the uncertainty on the damping time is expected to be large. Considering Fig. 2 of Wang \& Solanki (2004), we believe that a damping time of $714 \mathrm{~s}$ is rather long and that a damping time as short as $200 \mathrm{~s}$ may be consistent with the observational signature (T. J. Wang, private communication). A value for the density contrast has not been given.

Firstly, we consider the density perturbation. Using the observed values, the amplitude ratio $\mu=-14(-16 ;-12)$. Considering Fig. 1, we can see that the density contrast of the loop needs to be low $(\chi>0.5)$ and/or the value of $\alpha$ larger than 2 . This seems unlikely. In Paper I, we pointed out that the authors did not correct for the effect of time-integration in determining the amplitude of the intensity perturbation. Therefore, we do not know the exact value of amplitude of the relative average intensity perturbation for this observation.

In this example, $\alpha, \chi$ and $V_{\mathrm{Ai}}$ are all unknown and only measurements exist of $\mathrm{P}$ and $\tau$. Hence, the system is underdetermined. Figure 5 shows the $\alpha-\chi$ parameter space with the curves of constant $\tau / \mathrm{P}$ for two values of $\tau, 714 \mathrm{~s}$ and $200 \mathrm{~s}$. Along each curve the value of the Alfvén speed varies, increasing with decreasing $\alpha$. A few curves of constant $\mu$ are shown for illustration but cannot be used here for finding a crossing. Additional information is needed about $\chi$ or $\alpha$ to determine all unknown parameters. From the observations, the loop appears to be not well-contrasted. Possibly, this may be explained by the effects of line-of-sight integration and the narrowness of the temperature bandpass of the TRACE instrument. Nonetheless, it is reasonable to assume that $\chi$ would be larger than, say, 0.05 . This would mean that $\alpha$ is smaller than -2.8 and the Alfvén speed is larger than $700 \mathrm{~km} \mathrm{~s}^{-1}$.

Another approach is to assume a value of $\alpha$ and then determine the associated values for $\chi$ and $V_{\mathrm{Ai}}$ as follows. The wave period and damping time are calculated using dispersion relation (5) for a range of values in $\chi$ and compared with the observational counterparts using Eqs. (2) and (3). From each equation, the internal Alfvén speed is calculated. As a result, there are two independent relations between $V_{\mathrm{Ai}}$ and $\chi$. These relations form
Table 1. Parameters $\chi$ and $V_{\mathrm{Ai}}$ determined for three values of $\alpha$ and two values of $\tau$ and for which $a / R=0.03$. The range due to uncertainties is given between brackets.

\begin{tabular}{llll}
\hline \hline $\begin{array}{l}\tau \\
(\mathrm{s})\end{array}$ & $\alpha$ & $\chi$ & $\begin{array}{l}V_{\mathrm{Ai}} \\
\left(\mathrm{kms}^{-1}\right)\end{array}$ \\
\hline 714 & -3.5 & $0.027(0.008 ; 0.045)$ & $650(340 ; 830)$ \\
& -2 & $0.005(0.001 ; 0.008)$ & $540(280 ; 695)$ \\
0 & $0.001(0.0003 ; 0.002)$ & $520(270 ; 660)$ \\
200 & -3.5 & $0.095(0.028 ; 0.158)$ & $900(490 ; 1160)$ \\
& -2 & $0.030(0.008 ; 0.050)$ & $610(320 ; 780)$ \\
0 & $0.016(0.004 ; 0.025)$ & $530(280 ; 680)$ \\
\hline
\end{tabular}

curves in the $V_{\mathrm{Ai}}-\chi$ parameter space. Where the two curves cross, both relations are satisfied and a consistent solution of $V_{\mathrm{Ai}}$ and $\chi$ is found. We have followed this procedure for the average observed value $a / R=0.03$ and the two error range limits, for three values of $\alpha$ and for $\tau=714 \mathrm{~s}$ and $\tau=200 \mathrm{~s}$. Figure 6 illustrates the case with $\tau=200 \mathrm{~s}$. The values of $\chi$ an $V_{\mathrm{Ai}}$, determined for $a / R=0.03$, are shown in Table 1 . The large range associated with the determined values of $\chi$ and $V_{\mathrm{Ai}}$ is again due to the wide range in $a$. The obtained values are consistent with the procedure using the $\alpha-\chi$ parameter space with the relevant values of $\alpha$. As expected, the case of $\alpha=-3.5$ gives the most reasonable result compared with the other two chosen values. From the straight cylinder model of Edwin \& Roberts (1983), using values of $\chi$ equal to 0 and 0.1 , the Alfvén speed is estimated to be $2100-2200 \mathrm{~km} \mathrm{~s}^{-1}$. This is twice as large as the value of $900 \mathrm{~km} \mathrm{~s}^{-1}$ we found for $\alpha=-3.5$. A value of $\alpha$ closer to -4 would be needed to increase the speed.

\subsection{Example 2}

We consider the observations of transverse loop oscillations, as reported by Aschwanden et al. (1999), Nakariakov et al. (1999) and Aschwanden et al. (2002), for which measurements of the period and damping times exist. These events are considered to be horizontally polarised oscillation modes. Strictly speaking, our model is only valid for vertically polarised modes. For instance, the behaviour of the density perturbation is expected to differs between the two modes. However, because the observed oscillations are probably a superposition of both modes of 
Table 2. Comparison of observations of transverse oscillations with our model. The number refers to observations by Aschwanden et al. (2002); $V_{\mathrm{Ai}}^{(\mathrm{s})}$ is the internal Alfvén speed determined from the wave period using the straight cylinder model of Edwin \& Roberts (1983); $\alpha$ and $V_{\mathrm{Ai}}$ are the values of the density power law index and internal Alfvén speed consistent with the observations and our model. $\tau_{0}$ is the model predicted damping time for $\alpha=0$.

\begin{tabular}{lcrcccrcrc}
\hline \hline $\mathrm{Nr}$ & $\begin{array}{c}R \\
(\mathrm{Mm})\end{array}$ & $\begin{array}{c}a \\
(\mathrm{Mm})\end{array}$ & $\chi$ & $\begin{array}{c}P \\
(\mathrm{~s})\end{array}$ & $\begin{array}{c}\tau_{\mathrm{obs}} \\
(\mathrm{s})\end{array}$ & $\begin{array}{c}V_{\mathrm{Ai}}^{(\mathrm{s})} \\
\left(\mathrm{km} \mathrm{s}^{-1}\right)\end{array}$ & $\alpha$ & $\begin{array}{c}V_{\mathrm{Ai}} \\
\left(\mathrm{km} \mathrm{s}^{-1}\right)\end{array}$ & $\begin{array}{c}\tau_{0} \\
(\mathrm{~s})\end{array}$ \\
\hline $1 a$ & 47 & 3.5 & 0.20 & 261 & 870 & 880 & -3.86 & 520 & 110 \\
$1 b$ & 24 & 3.4 & 0.26 & 265 & 300 & 450 & -3.54 & 300 & 110 \\
$1 d$ & 55 & 2.6 & 0.27 & 316 & 500 & 870 & -3.81 & 550 & 100 \\
$1 f$ & 57 & 2.0 & 0.42 & 277 & 400 & 1090 & -3.87 & 780 & 60 \\
$1 g$ & 45 & 3.4 & 0.44 & 272 & 849 & 880 & -3.93 & 700 & 70 \\
$3 a$ & 99 & 12.4 & 0.32 & 522 & 1200 & 970 & -3.79 & 680 & 190 \\
$4 a$ & 74 & 2.8 & 0.14 & 435 & 600 & 810 & -3.75 & 400 & 180 \\
$5 c$ & 53 & 2.5 & 0.18 & 143 & 200 & 1790 & -3.76 & 870 & 60 \\
$10 a$ & 77 & 4.6 & 0.07 & 423 & 800 & 840 & -3.44 & 360 & 300 \\
$16 a$ & 68 & 10.2 & 0.32 & 185 & 200 & 1880 & -3.59 & 1300 & 70 \\
$17 a$ & 33 & 1.6 & 0.66 & 396 & 400 & 480 & -3.88 & 420 & 70 \\
\hline
\end{tabular}

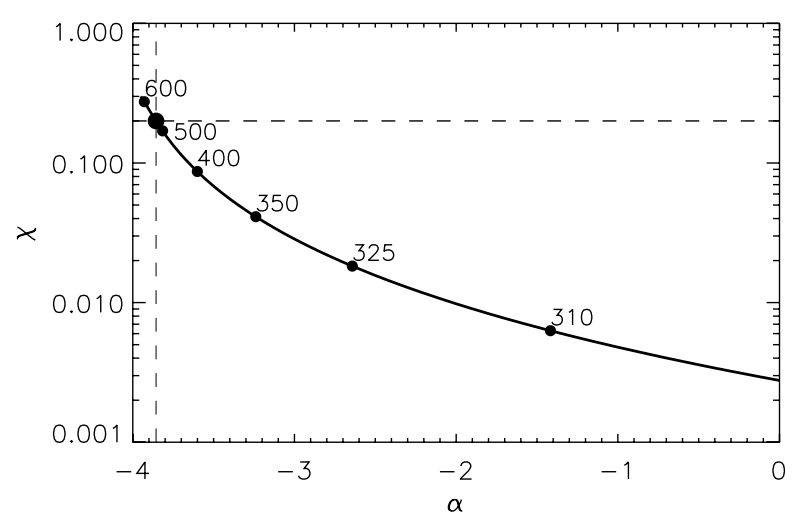

Fig. 7. Parameter space $\alpha-\chi$ for observation nr 1a from example 2 . The curve represents where the quality factor $\tau / P$, calculated using solution of Eq. (5), matches the observed value of 3.33. The aspect ratio $a / R=0.2$. Along these curves the value of $V_{\mathrm{Ai}}$ varies monotonically. Key values of $V_{\mathrm{Ai}}$ in units of $\mathrm{km} \mathrm{s}^{-1}$ are indicated. The dashed curves represent constant values of $\mu$.

polarisations, and lateral wave leakage may occur in the horizontal as well as the vertical direction, we shall use our model to make a preliminary assessment of the hypothesis that lateral wave leakage can explain the observed wave damping. Since Aschwanden et al. (2003) have measured the density contrast of the loops, only two parameters are unknown, i.e. $V_{\mathrm{Ai}}$ and $\alpha$.

Table 2 shows the observed loop and oscillation parameters, including the value of the internal Alfvén speed $V_{\mathrm{Ai}}^{(\mathrm{s})}$ as determined from the wave period using the straight cylinder model of Edwin \& Roberts (1983). The value of $\alpha$ is determined from where the curve of constant $\tau / P$ crosses the measured value of $\chi$ (see Fig. 7 for an example). $V_{\mathrm{Ai}}$ is consequently determined from the dimensionless frequency using Eq. (2). We find $\alpha$ to be in the range between -3.99 and -3.4 . Furthermore, we see that the values of the corresponding internal Alfvén speed are on average about $60 \%$ smaller than the values $V_{\mathrm{Ai}}^{(\mathrm{s})}$. This in turn would give values of the coronal loop magnetic field that are the same factor smaller than previously estimated.

In the two examples investigated here, we find a trend in that the parameter $\alpha$ needs to tend towards -4 for the model to be consistent with the observations. It seems unlikely that the density profile for all observed loops resemble each other so closely. Larger values of $\alpha$, say up to $\alpha=0$, seem plausible. Example 1 shows that for such values an unrealistically high loop contrast $(\chi<0.03)$ is needed in order to match the observed damping rate. For a more realistic density contrast, the model predicts too high a damping rate. Furthermore, example 2 shows that for $\alpha=0$, the model predicts damping times which are 3-10 times shorter than are observed (see Table 2).

\section{Discussion}

The mechanism of lateral wave leakage is efficient in damping vertically polarised fast kink oscillations, but our theoretical model predicts damping rates that are faster than the observations. However, this is not a reason for disqualifying this mechanism in explaining the observed damping. This rather points to the need for improving the model by way of a more realistic description of lateral leakage of fast kink oscillations. Hopefully, the theoretically predicted damping will then be closer to the observations. This may be achieved in several ways.

The slab geometry used in our model can be replaced by the more realistic cylindrical geometry. For a curved loop model this translates into a toroidal geometry with a two-dimensional equilibrium. We know, from straight coronal loop models, that the wave solution in the external medium has the form of an exponential for a straight slab and the form of a modified Bessel function for a straight cylinder. Because the modified Bessel functions decay faster with distance than exponentials, we expect the wave trapping to increase and therefore the efficiency of lateral leakage to decrease (e.g. Mikhalyaev \& Solov'ev 2005). Toroidal geometry also allows for the study of the effect of lateral leakage on vertically as well as horizontally polarised oscillations. The damping rate will then depend on the angle between the directions of polarisation and structuring of the Alfvén speed, e.g. a coronal loop with a vertically structured Alfvén speed profile will allow vertically polarised waves to leak out faster than horizontally polarised waves. The effect of lateral leakage of waves in coronal loops in toroidal geometry remains to be investigated. Van Doorselaere et al. (2004) studied fast magnetoacoustic kink oscillations in toroidal geometry, but restricted themselves to a linear Alfvén speed profile for which waves are trapped.

Also, a more realistic equilibrium density profile may be introduced, based on gravitational stratification, such that the density depends on vertical distance from the solar surface rather than radial distance from the centre of curvature of the loop (e.g. Andries et al. 2005; Mendoza-Briceño et al. 2004; Dymova \& Ruderman 2004). This would make the equilibrium twodimensional. We expect the rate of lateral leakage to become function of distance along the loop axis, which affects wave modes differently, depending on degree $m$.

Furthermore, we have modeled a coronal loop as an isolated structure. In reality, loops are part of groups of loops (e.g. arcades, fans). Therefore, besides wave modes of a single loop, a group of loops as a whole may support wave modes with different damping rates. The introduction of a sheared magnetic field is expected to introduce coupling between the Alfvén and magnetoacoustic modes (Goedbloed \& Halberstadt 1994) and it would be interesting to study this in a curved geometry.

Finally, it is useful to expand the model to take into account nonlinear effects. Although fast kink modes have nonlinear density perturbations that remain relatively small, they excite nonlinearly slow magnetoacoustic oscillations, which have density 
perturbations that can be comparable with those of linear fast kink oscillations in a curved loop (Terradas \& Ofman 2005). In modeling this, a finite plasma- $\beta$ needs to be taken to avoid unphysical results (see Verwichte et al. 1999, for a discussion). Also, as these perturbations act on a slower acoustic time scale, they do not oscillate in phase with the kink mode. Therefore, it should be possible to distinguish the different types of density perturbations from observations.

We have demonstrated how a model describing the mechanism of lateral wave leakage can be used for seismological purposes, providing information about the transverse loop structuring. Also, we stress that the method to determine the unknown model parameters is self-consistent, so that all observed and predicted wave parameters match. Lateral wave leakage is an attractive mechanism for explaining observed damping of fast kink oscillations and studies using more realistic models are warranted to explore the predicted damping rates.

Acknowledgements. The authors would like to thank the referee, Jesse Andries, for the many insightful comments which have greatly improved the paper. E.V. is grateful to PPARC for the financial support.

\section{References}

Andries, J., Goossens, M., Hollweg, J. V., Arregui, I., \& VanDoorselaere, T. 2005, A\&A, 430, 1109

Aschwanden, M. J., Fletcher, L., Schrijver, C. J., \& Alexander, D. 1999, ApJ, 520,880

Aschwanden, M. J., Schrijver, C. J., De Pontieu, B., \& Title, A. M. 2002, Sol. Phys., 206, 99

Aschwanden, M. J., Nightingale, R. W., Andries, J., Goossens, M., \& VanDoorsselaere, T. 2003, ApJ, 598, 1375
Brady, C. S., \& Arber, T. D. 2005, A\&A, 438, 733

Brady, C. S., Verwichte, E., \& Arber, T. D. 2006, A\&A, 449, 389

Dymova, M. V., \& Ruderman, M. S. 2005, Sol. Phys., 229, 79

Edwin, P. M., \& Roberts, B. 1982, Sol. Phys., 76, 239

Edwin, P. M., \& Roberts, B. 1983, Sol. Phys., 88, 179

Goedbloed, J. P., \& Halberstadt, G. 1994, A\&A, 286, 275

Goossens, M., Andries, J., \& Aschwanden, M. J. 2002, A\&A, 394, L39

Katsiyannis, A. C., Williams, D. R., McAteer, R. T. J., et al. 2003, A\&A, 406, 709

King, D. B., Nakariakov, V. M., Deluca, E. E., Golub, L., \& McClements, K. G. 2003, A\&A, 404, L1

Mendoza-Briceño, C. A., Erdélyi, R., \& Sigalotti, L. Di G. 2004, ApJ, 605, 493

Mikhalyaev, B. B., \& Solov'ev, A. A. 2005, Sol. Phys., 227, 249

Nakariakov, V. M., Ofman, L., DeLuca, E. E., Roberts, B., \& Davila, J. M. 1999, Science, 285, 862

Nakariakov, V. M., \& Ofman, L. 2001, A\&A, 372, L53

Nakariakov, V. M., Arber, T. D., Ault, C. E., et al. 2004, MNRAS, 349, 705

Nakariakov, V. M., \& Verwichte, E. 2005, Living Rev. Sol. Phys., 2, 3, URL (cited on 2005-07-05):

http: //www. livingreviews.org/lrsp-2005-3

Ofman, L., \& Aschwanden, M. J. 2002, ApJ, 576, L153

Roberts, B. 2000, Sol. Phys., 193, 139

Roberts, B., Edwin, P. M., \& Benz, A. O. 1984, ApJ, 279, 857

Robbrecht, E., Verwichte, E., Berghmans, D., et al. 2001, A\&A, 370, 591

Ruderman, M. S., \& Roberts, B. 2002, ApJ, 577, 475

Uchida, Y. 1970, Publ. Astron. Soc. Jpn., 22, 341

Terradas, J., \& Ofman, L. 2004, ApJ, 610, 523

Van Doorselaere, T., DeBosscher, A., Andries, J., \& Poedts, S. 2004, A\&A, 424, 1065

Verwichte, E., Nakariakov, V. M., \& Longbottom, A. W. 1999, J. Plasma. Phys., 62,219

Verwichte, E., Nakariakov, V. M., Ofman, L., \& DeLuca, E. E. 2004, Sol. Phys., 223, 77

Verwichte, E., Foullon, C., \& Nakariakov, V. M. 2006a, A\&A, 446, 1139

Verwichte, E., Foullon, C., \& Nakariakov, V. M. 2006b, A\&A, 449, 769

Wang, T. J., \& Solanki, S. K. 2004, A\&A, 421, L33 